\title{
High Altitude Wind Power Systems: A Survey on Flexible Power Kites
}

\author{
Mariam Ahmed* \\ Grenoble Electrical Engineering \\ Laboratory (G2ELab) \\ 38402 Saint-Martin d'Heres, \\ France
}

\author{
Ahmad Hably** \\ GIPSA-lab -ENSE3 BP 46 \\ 38402 Saint-Martin d'Heres, \\ France
}

\author{
Seddik Bacha*** \\ Grenoble Electrical Engineering \\ Laboratory (G2ELab) \\ 38402 Saint-Martin d'Heres, \\ France
}

\begin{abstract}
High altitude wind energy (HAWE) is a new field of renewable energy that has received an increasing attention during the last decade. Many solutions were proposed to harness this energy including the usage of kites, which were for a long time considered as child-toys. An overview on HAWE and its exploitation technologies is presented, followed by focusing on the the kite-based systems. Proposed structures and scenarios are presented, discussed and compared.
\end{abstract}

\section{INTRODUCTION}

Modern civilization is very energy dependent, especially on its nonrenewable sources, that will run out sooner or later. Using renewable energy sources instead of those nonrenewable is very promising but it is accompanied with a lot of scientific challenges. Because of this major interest in sustainable development, renewable energy systems, such as solar photo-voltaic, wind and tidal systems, are heavily explored.

One ideal source of renewable energy is the wind. Traditionally, wind turbines were used to exploit wind energy, they require no fuel and do not produce neither toxic nor radioactive wastes. Still, they are huge fixed constructions, and, although they undergo a lot of research and development to improve their efficiency, they are expected to reach their boundaries soon. A challenging limit is height; since higher altitude means stronger wind thus more kinetic energy [41]. At present, the highest wind turbine reaches $200 \mathrm{~m}$, where the wind is still unstable with an acceptable speed. In fact, strong wind could be present at higher altitudes with little or no wind at low altitudes. So, in order to overcome this classic wind-turbine limitation, new solutions to extract energy from high altitudes winds were investigated. This research led to thinking about investing kites in exploiting wind energy. This paper is an effort to collect and summarize the global work done during the last decade, on how kites (tetheredairfoils) are used to exploit high altitude wind energy (HAWE). After a brief discussion of the present global energy situation and its expectations, wind energy potential and classic wind turbines are argued. In section IV, HAWE exploitation systems are reviewed, followed by presenting the concept of power kites, which is the core of this paper, in section V. It includes explanation of how to harness wind power using a kite-based system, and the proposed structures

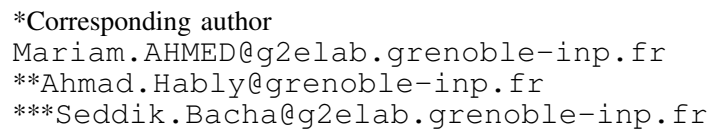

of such a system. In section VI, methods of controlling and optimizing kite-based systems are presented, and finally conclusions are made and further works are presented in section VII.

\section{Global Energy Situation and Expectations}

In order to understand the importance of investigating renewable energy resources and new ways to exploit them, it is necessary to consider the current global energy demands and production, and how they are expected to change in the next two decades. Fossil fuels that have been generated and stored underground since the birth of earth, which is estimated to be billions of years ago, have been heavily exploited during the last century. In fact, they are running out too fast, that the latest measurements confirm that the world's supply of oil and gas will not meet international demand at some time before 2020 [35]. Fig.1 shows the growth of different energy consumption for both OECD (Organization for Economic Co-operation and Development) and Non-OECD countries. It can be noted that Non-OECD economies are expected to be driving the consumption growth. In addition to limited

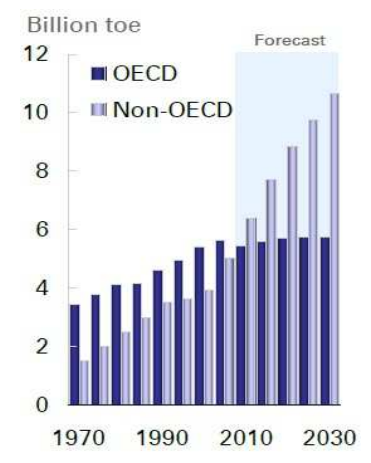

Fig. 1. Primary energy demands between 1990 and 2030 for OECD and Non-OCED countries [8]

future availability, fossil fuels are endangering the earth's climate, because of their $\mathrm{CO}_{2}$ emissions. The emissions that have already changed the climate dramatically during the last ten years due to global warming effect [9]. Fig.2 shows the amounts of $\mathrm{CO}_{2}$ emissions in both OECD and non-OECD countries due to fossil fuels. This danger threatening our earth has led to research replacing fossil fuels dependent machineries to electricity dependent ones. An electricity that is produced in a nature-friendly procedure.

The use of fossil fuels is a serious problem, not only 


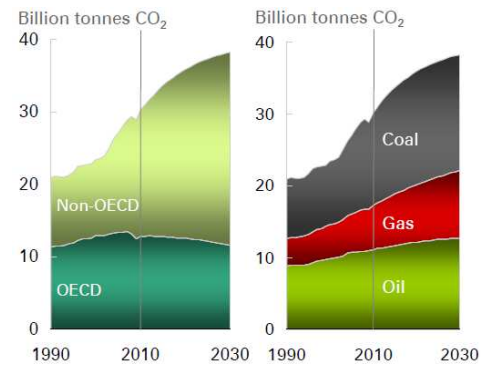

Fig. 2. $\mathrm{CO}_{2}$ emissions from energy use between 1990 and 2030 for: left, by region (OECD and Non-OCED), right, by fuel [8]

economically and climate wise; but on the humanitarian side, as its lack has and will enhance wars. Maybe it is somehow late to provide the needed energy using only nonfossil energy resources, but it worth working on to minimize the disastrous results of fossil fuels draining off.

One non-fossil energy source that was considered very promising for future energy, is nuclear energy. However, this source is facing many accusations considering its safety and cleanness. These accusations are now more intense after the recent Fukushima Daiichi nuclear disaster on March 2011 [48], which raised serious doubts about the future nuclear energy.

All mentioned points led to considering renewable energy resources, such as solar photo-voltaic, wind and tidal systems, as they offer the safest, cleanest type of energy. Extensive studies have been done on how to extract energy from natural renewable resources and how to transform and inject it in the electric grid. Still, as shown in Fig.3, the growth of exploited renewable energy is not that impressive compared to other resources, which means more investments and awareness on this field should be achieved, mainly in the Non-OECD countries.

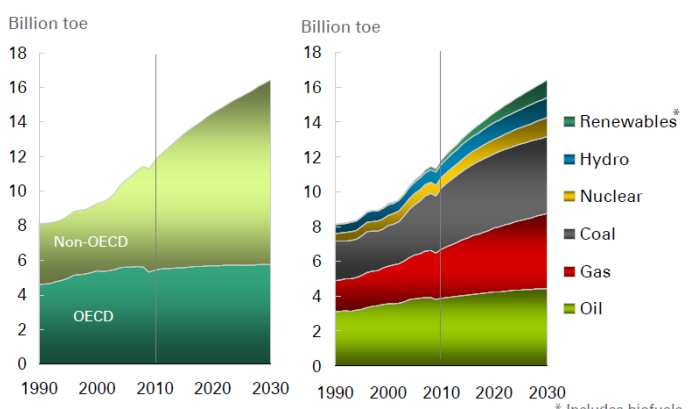

Fig. 3. Energy demands between 1990 and 2030 for: left, by region (OECD and Non-OCED), right, by energy resources [8]

\section{WIND ENERGY}

Wind is considered an ideal renewable energy resource, since it is infinitely sustainable and clean. This explains the global interest in exploiting its energy; actually, between 2000 and 2009, wind energy global capacity increased more than nine-fold, growing from 17.4 GW to $158.6 \mathrm{GW}$ [17]. That makes wind, not only the fastest growing renewable energy resource, but also the fastest growing electric power resource of all [13].

A conventional wind turbine is a two/three-blade rotor, that converts wind's kinetic energy into electrical energy. This is done by a direct, or through a gearbox, coupling with an electric generator. The turbine is installed on the standard electric grid through a power-electronics interface. The turbine power is controlled by commanding the pitch and yaw blades angles. The whole conversion chain and the blades' control unit are placed up next to the rotor hup, on the turbine's tower. As for turbines size and rated power, in 2011, Enercon has presented its E-126 wind turbine whose rotor diameter reached $126 \mathrm{~m}$, a tower height of $135 \mathrm{~m}$; and a total mass of 6,000 tons, this turbine's rated power is 7.5MW. One aim of increasing turbines size is to reach higher altitudes where winds are supposed to be stronger and more stable. In fact, the amount of wind energy available for extraction increases with the cube of wind speed [41]. The other objective is to increase the blades size thus the turbine working area, with which the available wind power increases linearly.

Nevertheless, turbine size is not expected to grow as dramatically in the future as it has in the past [49]; for the cost, which includes manufacturing, transportation and construction, at some point starts growing faster than the resulting rated power [23], as well as, turbines are not able to produce their rated power continuously, due to wind irregularity. To overcome these limitations, new axes of research have started. One aims at making use of the strong off-shore wind, and has led to "Off-shore turbines", while the other axis looks for structures able to extract wind energy at high altitudes. The latter is focused on in this paper, more specifically, systems that use flexible kites for exploiting wind energy are considered. An example to compare a kite-based system to a classic turbine is given in [23]: A $2 M W$ wind turbine, the weight of the rotor and the tower is typically about 300 tons, while a high altitude generator of the same rated power is estimated to be obtained using a $500 \mathrm{~m}^{2}$ airfoil and $1000 \mathrm{~m}$ long cables, with a total weight of about 2 tons only.

\section{High Altitude Wind Energy Technologies}

Several designs are proposed to harness HAWE such as balloons, airborne wind turbines and tethered airfoils (kites). The principle of the balloon, developed by Magenn Power Inc, is as follows: a helium-filled balloon stationary at a height between $200 \mathrm{~m}$ and $350 \mathrm{~m}$ rotates around a horizontal axis in response to wind, generating electrical energy by a generator connected to its horizontal axis. The energy produced is then transmitted to the ground by a conductive tether. The second solution offered by Sky WindPower, Joby energy [30], or Makani Power [31] would be to use airborne wind turbines to harness energy directly in high altitude winds and to send it to the ground by cables. This system has some technical complexities and high cost with a significant risk related to its weight. The third option is to use power kites as renewable energy generators such as the "Kite Wind 
Generator" of Politecnico di Torinoand "Laddermill" of the Delft University of Technology.

\section{Flexible Power Kites}

Kites were used in China approximately 2,800 years ago. Apart from being child-toys, their early uses involved measuring distances, testing the wind, lifting men, signaling, and communication for military operations. After spreading in Asia, kites were brought to Europe by Marco Polo at the end of the 13th century [6]. In the 18th and 19th centuries, in addition to military operations, kites were used to tow buggies in races against horse courages in the country side [27], and researchers like Alexander Graham Bell and the Wright Brothers invested kites in their development of the airplane. At the beginning of the 20th century, interest in kites was diminished by the invention of airplane. Kites drew back attention in 1972 when Peter Powell introduced a steerable dual lines kite, and flying kites became a sport. The idea of exploiting wind energy using kites, came up when Loyd discussed the ability of a crosswind flying kite to generate up to 5 times the energy generated by an equivalent fixed turbine [40]. Though his paper was completely ignored at the time, it is now considered a revolutionary idea, that can contribute hugely in renewable energy production.

Nowadays, few groups around the world are working on this concept, including the Kitegen project launched in 2007 by a research team from Politecnico di Torino in Italy, they already built and tested a prototype but their research has slew down recently due to lack of investment. Other university research teams are in K.U.Leuvent in Belgium, T.U.Delft in Netherlands, the university of Heidelberg. in Germany; and the university of Grenoble in France. These teams are focusing on the theoretical aspect of how to model, control and optimize the performance of a kite-based system. Using flexible kites for towing ships is already in the market by Skysails GmbH company in Hamburg, Germany [3]. Installing kites on huge ships proved to reduce their fuel consumption up to $30 \%$. Some other companies names in the field include: Makani Power company in the USA [31], Windlift [5], SwissKitePower [4], and Worcester Polytechnic Institute (WPI) project \#DJO-0408 [19].

The structure and control strategy adapted by each team, as well as a comparison between them, will be presented in the course of the coming paragraphs.

\section{A. Crosswind Kite Power}

In his paper [40], Loyd analyzed three ways by which the kite can generate energy. Two are significantly more effective:

- The Drag power, that can be exploited by having air turbines on the kite. The concept was employed in the airborne wind turbines of Joby energy [30], the balloon of Megenn Power Inc [2], the Skymill [7]; and the "Makani M1" which is a $1 M W$ Airborne wind turbine built by Makani Power Company [31].

- The crosswind power, by neglecting the kite's weight and the cable's weight and drag force, and considering the kite motion to be directly in crosswind ${ }^{1}$; then the kite's speed is increased above the wind speed, which leads to increasing the can-be-generated power.

The second concept is the one investigated here, however, it is not possible to let the kite fly always in a crosswind direction. In [14], Argatov has derived the refined crosswind motion low of eq. 1 that takes into account the kite's weight and the cable influence.

$$
\left|W_{e}^{p}\right|=G_{e} V_{\|}, \vec{W}_{e}^{p}=\vec{W}_{e}-\left(\vec{e}_{r} \cdot \vec{W}_{e}\right) \cdot \vec{e}_{r}
$$

where $\vec{W}_{e}$ is the effective wind speed, $\left(\vec{e}_{r} \cdot \vec{W}_{e}\right) \cdot \vec{e}_{r}$ is its projection on the cable direction, $V_{\|}$is the crosswind speed and $G_{e}$ is aerodynamic efficiency.An eight-shaped trajectory of the kite is highly adapted, since it maximizes the apparent wind blowing against the kite, and ensures the non-tangling of its cables. But it should be mentioned that, according to [29], a circular trajectory will provide a $0.9 \%$ to $1.3 \%$ higher traction force than that of an eight-shaped one, in addition to ignoring the wind direction that may affect the system's safety and control [12]. Still, cables tangling and coiling remains serious problem.

\section{B. Energy Generation Concept}

The concept of a kite-based system is to mechanically drive a ground-based electric generator using one or several tethered kites. Energy is extracted from high altitudes by controlling the kite to fly with a high crosswind speed. This develops a large pulling force that turns the generator, thus generating electricity. However, due to limitation in the tether's length and the power region, the kite should always be redrawn to its initial position, consuming energy as doing so. In general, three types of the kite-based system were adapted:

1) The Pumping System: ${ }^{2}$ It is largely adapted by several research teams. The system has two operation phases. A traction phase, in which the kite is pulled by the wind, unrolling the cable which turns the ground-based machine; and a recovery phase, that begins when the cable reaches its predefined maximum length, and needs to be rewound, an operation that consumes energy. To minimize this consumption the KiteGen project has presented two methods [24]:

- Low power recovery maneuver: The kite is driven to the borders of the power zone, where it can be recovered with low energy expense. One down point of this maneuver is that it occupies a huge space, which is a problem when it comes to considering a kite-farm for example.

- Wing glide maneuver: Here the kite is controlled to be parallel to the tether, thus it loses its aerodynamic lift and can be wound back fast with low energy losses. Nonetheless, this maneuver, subject the system to instability and difficulty to restart its cycle.

\footnotetext{
${ }^{1}$ This means the cable is parallel to the wind.

${ }^{2}$ Referred to as the yo-yo mode of KiteGEN, or the open-loop orbit of Argatov.
} 
Sequent eight-shaped orbits with increasing altitude are usually adapted in the traction phase [16], [37], [46], [28], [22]. Apart from this, another idea started in SEQUOIA Company, directed by Gianni Vergnano, is the Rotokite. The idea proposes, as shown in Fig.4, having two opposed kites connected to a single cable. They are controlled to rotate around the cable, generating a lift force that will pull out the cable (traction phase), and when the maximum height is reached, they are warped to minimize the aerodynamic lift, and then pulled down. This idea is now a part of Google project $10^{100}$ due to lack of funding.

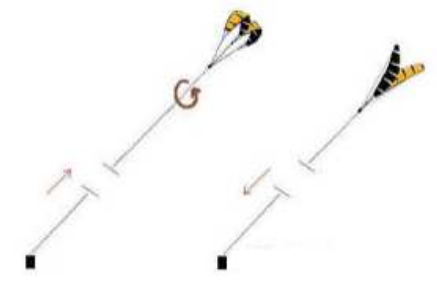

Fig. 4. Rotokite operation principal [50].

2) The Closed-Orbit System: The kite is kept on a single eight-shaped orbit. During one orbit, two regions can be distinguished: A high and a low crosswind region. In the high crosswind region, the kite pulls out the cable which forms the "traction phase", and in the low crosswind region, the cable is wound in, and that is the "recovery phase". This mode may not generate as much energy as the pumping mode [15], but it is easier to be stabilized and controlled, as it needs one controller only compared to at least two controllers in the pumping mode, as well as it occupies a small space [39]. Moreover, its production can be increased by adding the possibility to control the aerodynamic coefficients by the kite's attack angle [15].

3) The Carousel System: This system, proposed by M.Ippolito [34], suggests placing several tethered-kites with their control units on vehicles moving along a circular rail path. This idea is being invested in the carousel KIWIGen project, for which Fig. 5 shows a simplified presentation.

Vehicles' speed is kept constant using electric machines on their wheels, while the kites tether's length might be fixed or have a rolling/unrolling motion [22]. For the first case, two phases are distinguished, a traction phase, during which the kite pulls the vehicle while it is in the power zone, and a recovery phase where the vehicle moves the kite back to the power zone. According to [25], this complex structure generates the same power that can be produced using a simple pumping kite.

Although by applying a rolling/unrolling (pumping) motion of the kite to compensate the consumed energy during the recovery phase, a highly efficient system can be obtained, it is still economically and technically not feasible.

\section{Kite-Based System Structure}

As presented in Fig.6, a simplified kite-based system consists of a kite connected to a drum by a cable. The

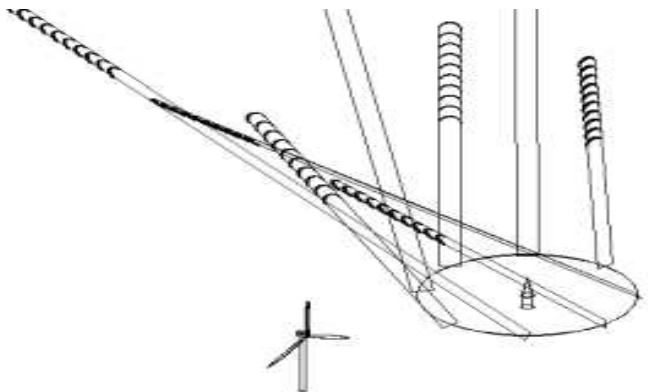

Fig. 5. Simplified presentation of the carousel KIWIGen project.

drum changes the traction force generated by the kite into a resistive torque applied on an electric machine through a gearbox. The machine converts this mechanical traction to an electrical energy that can be later stored or injected in the electric grid.

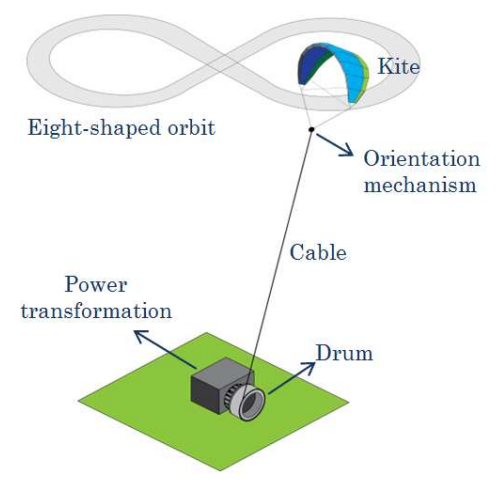

Fig. 6. A simplified kite-based system.

In the next paragraphs, important aspects of a kite-based system are presented in more details.

1) The Kite: In order to operate, the kite must be in its power zone, a zone where enough lifting is applied on it. It is a half hemisphere, whose center is the point where the cable is parallel to wind direction.

Just like an airplane wing, a kite is characterized by two main parameters: Its aspect ratio $A R$ and its aerodynamic efficiency $G_{e}$. The aspect ratio is the ratio between the kite span $w_{s}$ and chord $c$ (see Fig.7-(a)). Meanwhile, the kite efficiency is the ratio between its lift and drag coefficient $\left(C_{L}, C_{D}\right)$. These coefficients are functions of the kite's attack angle $\alpha$, which is the kite angle with the effective wind $W_{e}$. The forces acting on the kite are (see Fig.7-(b)):

- $F^{\text {grav }}$ the gravity force

- $F^{a p p}$ the apparent force which is a result of the kite's acceleration.

- $F^{a e r}$ the aerodynamic force which has two components (eq.2): The lift force $F_{L}$ that is perpendicular to the kite's surface $A$, and the drag force $F_{D}$ which has the effective wind's direction $\vec{W}_{e}$.

$$
F_{L}=\frac{1}{2} \rho_{a} A C_{L}\left|\vec{W}_{e}\right|^{2}, \quad F_{D}=\frac{1}{2} \rho_{a} A C_{D}\left|\vec{W}_{e}\right|^{2}
$$


where $\rho_{a}$ is air density and $A$ is the kite surface. - $F^{c, t r c}$ is the traction force of the tether.

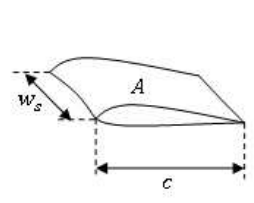

(a)

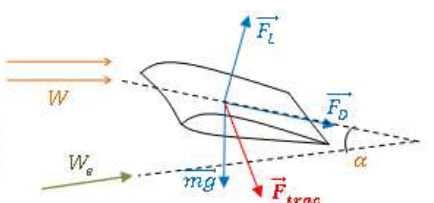

(b)
Fig. 7. Kite's main parameters and forces.

The interest is to have a maximum traction force, which means a high aerodynamic force and a low gravity force. So in order to use a specific kite in a wind energy exploitation system, it should satisfy a few criteria. Most importantly, it should have a high aerodynamic efficiency, and it should be light but still strain, resistive; and easily maneuvered.

Being light and strain depends basically on the materials the kite is made up of. A high aerodynamic efficiency and a maneuverability, both depend on the kite design, but each has opposite requirements to the other [24]. So a kite designer should find a trade-off between these two.

Another important parameter is the kite surface. On the positive side, the kite's exploited power is a linear function of the surface [36], as well as, a bigger surface enables the kite to work at higher wind speed, and results in more stability. On the negative side, the biggest the kite is, the more difficult it is to be oriented.

When first was suggested, the laddermill [43] proposed using rigid wings, but due to safety issues [44], they were soon changed by flexible light-weighted kites. Suggestions are made to improve flexible kites' efficiency by using multiple kites on the same cable (or set of cables). Fig. 8 shows two proposed structures. The first is to have a stack of kites, for instance the laddermill of Delft [39]. The second is to link two kites to the end of the same cable, which is useful to reduce the cable drag and the power needed in the recovery phase [29]. Both described structures will allow having the same traction force for a smaller space and less land requirements, but are more difficult to be modeled and controlled.

For early experiments, commercial kites were used, such as

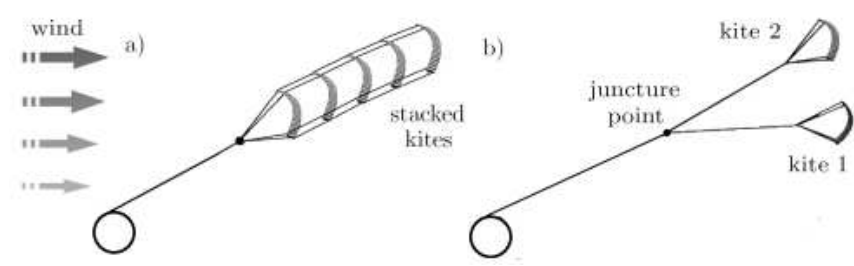

Fig. 8. Multiple-kite proposed structures [29].

the clark-y kite $\left(10 \mathrm{~m}^{2}\right)$ used in KiteGEN program [21], the Peter Lynn Guerilla $\left(10 \mathrm{~m}^{2}\right)$ Kite used by Worcester Polytechnic Institute (WPI) team [36], and the Peter Lynn Bomba $\left(8.5 \mathrm{~m}^{2}\right)$ surf-kite used by Delft team for their smallscale $2 k W$ testing laddermill [38]. However, much bigger and more specialized kites must be designed and tested in the future.

2) The Cables: Cables are needed to transfer the Aerodynamic forces acting on the kite to a traction force acting on the ground-based generator. Two aspects are important here, the number of cables, and their design, including their diameter and composition.

The number usually varies from one to five cables. The less the cables are, the more difficult it is to control the kite's orientation by earth-based controllers. On the contrary, according to [36], using more than one-cable scenario decreases the system efficiency. However, the KiteGEN and WPI team [19] have chosen to use 2 and 4 cables respectively, as this allows them to avoid the need to have controllers installed on the kite, thus having heavier kites, in addition to problems associated with wireless communication between the controllers and the on-ground control unit.

Furthermore, cables should be strong enough to bear high tractions, but light and with a small diameter in order to neglect their gravity and drag force $F_{t}$. The KiteGen used, for its small scale prototype, $1000 \mathrm{~m}$ cables made up of composite materials (Dyneema), that make it as light as a similar steel cable but 8 to 10 times more resistive [23].

For large scale kite-based systems, the cable model should be considered when controlling the system. A realistic model will take into account the cable weight and drag force, and includes its elasticity [20], [45].

3) Wind Speed Estimation: An important issue to consider when it comes to a HAWE system, is to estimate the wind speed. This can be done by having on-board observers, or, and since the wind is supposed to be stable at high altitudes, it can be calculated using a wind-speed model. Many models are listed in [13], but two main models are used in the literature [32] to know the wind speed $V$ at an altitude $z$;

$$
V(z)=v_{0}\left(\frac{z}{z_{0}}\right)^{\gamma}, \quad V(z)=v_{0} \frac{\ln \left(\frac{z}{z_{r}}\right)}{\ln \left(\frac{z_{0}}{z_{r}}\right)}
$$

where $v_{0}$ is the measured wind speed at an altitude $z_{0}$, and $\gamma$ is a friction coefficient that characterizes the surface above which the measurement is done, and can be described by the roughness factor $z_{r}$.

Previous models of the kite, the cables and the wind are employed to simulate the kite-based system, and test proposed control strategies.

\section{Kite-BASEd System CONTROL}

Generally, a kite-based system control should, on one hand, ensure the kite's (or kites set) tracking of a certain trajectory that respects the systems constraints, and on the other hand, manage the power generated/consumed by the system. For this reason, two aspects are considered: the control strategy and power management.

But first, how is the reference trajectory chosen? Two possibilities are available. Either by choosing an eight-shaped ${ }^{3}$

\footnotetext{
${ }^{3}$ For its wide application, an eight-shaped trajectory is the one focused on.
} 
orbit and optimize its period and velocity to have a maximum power generation, as suggested by Argatov [15], and used in [33] and in [51], or by maximizing directly the generated power while following an imposed eight-shaped path that respects the state constraints as KiteGEN team has done [24].

\section{A. Control Strategy}

The control unit should insure a stable behavior of the kite that overcomes weather changes, and to direct the kite to follow a certain trajectory. A general model of the system has the form,

$$
\dot{x}(t)=f(x(t), u(t), P(t))
$$

with constraints on the state $x(t)$ and the control vector $u(t)$,

$$
x_{\text {min }}<x(t)<x_{\max }, \quad u_{\min }<u(t)<u_{\max }
$$

The state vector $x(t)$ contains information on the kite's position and velocity, usually presented in the spheric coordinates. Meanwhile, the control vector $u(t)$ may contains controls of the kite different angles: roll, yaw and attack angle, in addition to control of the cable length variation rate. As for ' $P(t)$ ', contains all the external effects that influence the system behavior, e.g. wind perturbations. Depending on the system design and the measurements sensors, the control strategy is built, or vice versa.

Measuring the state vector is done through use of observers or sensors such as GPS receivers or Gyro sensors connected to the ground control unit through wireless communication. The mentioned constraints include, the kite flying in a limited area and avoiding being crashed, not exceeding a certain velocity or lift force, specified by the kite's and cable's properties and respects the ground-based electric machine rotating velocity, and constraints on the control variables and their changing rates.

As the kite-based system is nonlinear, constrained, and unstable in the open-loop, an equally complex sophisticated autonomous control strategy should be applied, with a sufficiently small sampling time to insure the tracking of any possible disturbance.

A simple linear control [51] is not suitable to control such a system as it cannot handle disturbances. It is only efficient around the linearizing point. A nonlinear model predictive control (NMPC) has proved to be convenient to generate the needed controls for trajectory tracking, though, [18] doubted the performance of this method, because it leads the solution to converge to different local optima depending on the initial conditions, and consume a lot of memory when it comes to application. Still NMPC was used in [33], [22], [11]. Other trajectory tracking suggested methods include neural network control [26], robust control [47], direct-inverse control [42]; and nonlinear adaptive tracking control [18].

Two examples of control strategies are mentioned here. In T.U.Delft, a one-cable kite control is tested in simulation; the kite surface is $25 \mathrm{~m}^{2}$ with a mass of $50 \mathrm{~kg}$ and the cable average length is $1000 \mathrm{~m}$. An optimal control is used to command the roll angle, attack angle, and the cable length variations rate. It uses different random guesses because there is no guarantee that the obtained solution is a global one. The average power generated depends on the orbit period, for example, a $60 \mathrm{sec}$ period orbit yields in $75 \mathrm{~kW}$ [52].

The KiteGEN team has chosen a two-cable kite for their prototype, with control of the cable length variations rate and the roll angle. For simulation, a kite of $10 \mathrm{~m}^{2}$ and a mass of $4 \mathrm{~kg}$; and a maximum $800 \mathrm{~m}$ long cables. A nonlinear predictive control, without a pre-computed trajectory, is applied. It maximizes the average generated power directly. Besides, a sampling time of $0.2 \mathrm{sec}$ is obtained by applying a Fast NPC, for the sake of real time control computations. With a windspeed of about $7.7 \mathrm{~m} / \mathrm{s}$ at $300 \mathrm{~m}$ altitude, the average generated power is $5 \mathrm{~kW}$ [23].

\section{B. Power Management}

Depending on the kite-based system structure, the system may be a power generator all the time, or a periodically producer/consumer. An example of the first kind is proposed in [36]. It involves connecting two kites to the same generator, and control them contrarily, in order to have a positive energy all the time. Another example is the carousel configuration with variable cable length (see sectionV-B.3).

Power management is important when the system is generator/consumer and connected to an isolated grid. In this case the system should store during the generator phase enough energy to continue supplying the grid and provide the needed energy to recover the kite initial position. while if the system is installed on an infinite electric grid, the system generates its maximum energy and injects it in the grid, and the grid will insure the needed energy for the recovery phase [10].

\section{CONCLUSIONS AND PERSPECTIVES}

\section{A. Conclusions}

In the search for alternatives to fossil fuel energy, high altitude wind energy (HAWE), and more specifically Kites or airborne wind energy (AWE), are receiving an increasing interest at present. Many technologies are proposed and intensive studies of ways to control them are going on. In addition to introducing the concept of "HAWE", and the cross wind energy, the paper tries to sum up, to the authors knowledge, what is done until now in this field of interest, and to come over the groups working on it, by presenting and comparing their approaches. It was shown, that these approaches are still in the simulation and small prototypes phases, but are expected to reach the application phase soon due to intensive work, interest and awareness of many research teams and sponsors.

\section{B. Perspectives}

A recent questionnaire following the Airborne Wind Energy Conference AWEC 2011, entitled: "Delphi analysis on airborne wind energy" summed up the expectations, problems and challenges facing kites or airborne based systems. The results show a special interest in the pumping or yo-yo concept. By 2015, experts expect to start marketing fully autonomous controlled kite-based systems, including start/land automation. These systems are expected to utilize 
$75 \%$ of their capacity by 2021 . Some problems and challenges include public acceptance, safety and performance issues, grid connection [10], in addition to competitive costs compared to the conventional wind turbines as well as other renewable energy sources [1].

This paper was written to provide a base for a research going on in G2ELAB/GIPSA Laboratories in Grenoble, France, that aims at controlling and studying the possibility of integrating a limited-cycle system, such as the proposed kite-based system on an isolated or infinite electric grid.

\section{REFERENCES}

[1] http://www.gl-garradhassan.com/en/highaltitudewind.php.

[2] http://www.magenn.com/.

[3] http://www.skysails.info.

[4] http://www.swisskitepower.ch.

[5] http://www.windlift.com.

[6] Kite history: A simple history of kiting, http:// www.gombergkites.com/nkm/hist1.html.

[7] Skymill: An innovative new approach to large-scale wind power. Industrial fuels and power, 2011.

[8] BP statistical review of world energy. London, United Kingdom, June 2010.

[9] International Energy Agency. $\mathrm{CO} 2$ emissions from fuel combustion highlights. 2011.

[10] M.S. Ahmed, A. Hably, and S. Bacha. Grid-connected kite generator system: Electrical variables control with MPPT. Industrial Engineering Conference, Melbourne, Australia, November 2011.

[11] M.S. Ahmed, A. Hably, and S. Bacha. Power maximization of a closed-orbit kite generator system. In 50th Conference on Decision and Control Conference (IEEE CDC), Orlando, USA, 2011.

[12] C.L. Archer and K. Caldeira. Global assessment of high-altitude wind power. Energies, 2(2):307-319, 2009.

[13] C.L. Archer and M.Z. Jacobson. Evaluation of global wind power. Journal of Geophysical Research, vol.110, D12110, 2005.

[14] I. Argatov, P. Rautakorpi, and R. Silvennoinen. Estimation of the mechanical energy output of the kite wind generator. Renewable Energy, 34:1525-1532, 2009.

[15] I. Argatov and R. Silvennoinen. Energy conversion efficiency of the pumping kite wind generator. Renewable Energy, 35(5):1052-1060, 2010 .

[16] I. Argatov and R. Silvennoinen. Structural optimization of the pumping kite wind generator. Structural and Multidisciplinary Optimization, 40(1):585-595, 2010.

[17] American Wind Energy Association (AWEA). U.S. wind industry annual market report: year ending 2009. Washington, D.C., 2010.

[18] J. H. Baayen and W.J. Ockels. Tracking control with adaption of kites. IET control theory \& applications, 6(2):182-191, 2011.

[19] G. Baldwin. Kite Power for Heifer Internationals Overlook Farm. PhD thesis, Worcester Polytechnic Institute, 2008. URL: http://www.energykitesystems.net/O/WPI/index.html.

[20] J. Breukels and W.J. Ockels. A multi-body dynamics approach to a cable simulation for kites. In Proceeding of the IASTED Asian Conference on Modelling and Simulation, pages 168-173. ACTA Press, 2007.

[21] M. Canale, L. Fagiano, and M. Milanese. Power kites for wind energy generation, fast predictive control of tethered airfoils. IEEE Control Systems Magazine, pages 25-38, 2007.

[22] M. Canale, L. Fagiano, and M. Milanese. High altitude wind energy generation using controlled power kites. IEEE Transactions On Control Systems Technology, 18(2):279 - 293, 2010.

[23] L. Fagiano. Control of Tethered Airfoils for High Altitude Wind Energy Generation. PhD thesis, Politechnico di Torino - Doctoral School, 2009. URL: http://lorenzofagiano.altervista.org/.

[24] L. Fagiano, M. Milanese, and D. Piga. High-altitude wind power generation. Energy Conversion, IEEE Transactions on, 25(1):168180, 2010.

[25] L. Fagiano, M. Milanese, and D. Piga. Optimization of airborne wind energy generators. International Journal of Robust and Nonlinear Control, 2011.

[26] A. Furey and I. Harvey. Evolution of neural networks for active control of tethered airfoils. Advances in Artificial Life, pages 746-755, 2007.
[27] Saul Griffith. Saul griffith on kites as the future of renewable energy. TED Talks, 2009.

[28] B. Houska and M. Diehl. Robustness and stability optimization of power generating kite systems in a periodic pumping mode. In IEEE Multi - Conference on Systems and Control, pages 2172-2177, 2010.

[29] B. Houska and M. Diehl. Optimal control of towing kites. In 45th IEEE Conference on Decision and Control, pages 2693-2697, San Diego, CA, USA 2006.

[30] http://www.jobyenergy.com/.

[31] http://www.makanipower.com/.

[32] M. Hussain. Dependence of power law index on surface wind speed. Energy conversion and management, 43(4):467-472, 2002.

[33] A. Ilzhofer, B. Houska, and M. Diehl. Nonlinear MPC of kites under varying wind conditions for a new class of large-scale wind power generators. International Journal Of Robust And Nonlinear Control, 17:1590-1599, 2007.

[34] M. Ippolito. Vertical axis wind turbine with control system steering kites, February 27 2008. EP Patent 1,672,214.

[35] CNN's Graham Jones. World oil and gas 'running out'. 2005 Cable News Network LP, LLLP., 2 October 2003.

[36] J. Krenciszek et al. Mathematical modeling of the pumping kite wind generator: Optimization of the power output. In URL: http://www.win.tue.nl/casa/meetings/special/ecmi08/pumpingkite.pdf, 2008.

[37] B. Lansdorp and W.J. Ockels. Comparison of concepts for highaltitude wind energy generation with ground based generator. In Proceedings of the NRE 2005 Conference, Beijing, China, pages 409417, 2005.

[38] B. Lansdorp, B. Remes, and W.J. Ockels. Design and testing of a remotely controlled surfkite for the laddermill. In World Wind Energy Conference, Melbourne, Australia, 2005.

[39] B. Lansdorp and P. Williams. The laddermill - innovative wind energy from high altitudes in holland and australia. In Windpower 06, Adelaide, Australia, 2006.

[40] M.L. Loyd. Crosswind kite power. J. ENERGY, 4(3), 1980.

[41] G.M. Masters. Renewable and Efficient Electric Power Systems. John Wiley \& Sons, Inc., Hoboken, New Jersey., 2004.

[42] C. Novara, L. Fagiano, and M. Milanese. Direct data-driven inverse control of a power kite for high altitude wind energy conversion. In IEEE International Conference on Control Applications (CCA), pages 240-245, 2011.

[43] W.J. Ockels. Patent 1004508, 12 November 1996

[44] W.J. Ockels. Laddermill, a novel concept to exploit the energy in the airspace. Aircraft Design, 4:81-97, 2001.

[45] A.R. Podgaets and W.J. Ockels. Three-dimensional simulation of a laddermill. In Proceedings of the 3rd Asian Wind Power Conference, Beijing, China, 2006.

[46] A.R. Podgaets and W.J. Ockels. Flight control of the high altitude wind power system. In Proceedings of the 7th Conference on Sustainable Applications for Tropical Island States, 2007.

[47] A.R. Podgaets and W.J. Ockels. Robust control of laddermill wind energy system. In Proceedings of the Wind Power Shanghai Conference, China, 2007.

[48] Eliza Strickland. Explainer: What went wrong in japan's nuclear reactors. IEEE Spectrum, 13 May 2011.

[49] R. Thresher, M. Robinson, and P. Veers. To capture the wind. IEEE power \& energy magazine, pages 34-46, 2007.

[50] G. Vergnano. High altitude wind generation: The rotokite. SEQUOIA IT.

[51] P. Williams, B. Lansdorp, and W. Ockels. Modeling and control of a kite on a variable length flexible inelastic tether. In AIAA Guidance, navigation and control conference, South Carolina, USA, 2007.

[52] P. Williams, B. Lansdorp, and W. Ockels. Optimal cross-wind towing and power generation with tethered kites. In AIAA Guidance, Navigation and Control Conference and Exhibit, 2007. 